\title{
Estimation of Species Area Abundance from Point Abundance Data, Using Effective Detection Areas from Camera Traps
}

Rademaker $\mathbf{M}^{1,2^{*}}$, Rode-Margono $\mathrm{EJ}^{\mathbf{3}}$ and Weterings $\mathrm{MJA}^{4}$

${ }^{1}$ UUCN/SSC Wild Pig Specialist Group, Jakarta, Indonesia

${ }^{2}$ Forest and Nature Conservation Program, Wageningen University, Wageningen, The Netherlands

${ }^{3}$ The North of England Zoological Society / Chester Zoo, Chester, United Kingdom

${ }^{4}$ Resource Ecology Group, Wageningen University, Wageningen, The Netherlandsp

\begin{abstract}
Estimations of species abundance are a common goal of wildlife monitoring surveys, but debate remains as to which methods are theoretically and practically most useful. Abundance-induced heterogeneity (AlH) models developed in the early 2000s allowed estimation of point abundance from repeated presence-absence data (e.g. occupancy models), and advanced estimation of point abundances of unmarked species. AlH models, however, do not provide an estimate of the effective detection area sampled. Therefore the absolute number of individuals in a survey area cannot be estimated directly. Recently, methods have become available to determine the effective detection area sampled by camera traps. Our objective was to present a novel method to estimate the absolute number of individuals of a species in an area from point abundance data using effective detection areas from camera traps. This would make AlH models available for population estimates. We applied this newly developed Species Area Abundance (SAA) model to a 3-month camera trapping data set of Bawean warty pigs (Sus blouchi) from Indonesia, and compared the result to an independent Random Encounter Model (REM) estimate from the same data. Population sizes and uncertainties estimated by the SAA and the REM model were comparable. Differences in density estimations between the REM and SAA model were not significant when mean group size was included in the REM. The less restrictive assumptions regarding camera trap placement of the SAA model compared to the REM might make it more practical to study cryptic and unmarked animal populations. Further studies are needed to determine the accuracy and practicality of the SAA model using a range of differrent sampling designs and focus species.
\end{abstract}

Keywords: Abundance-induced heterogeneity model; Bawean warty pig; Occupancy; Activity; Random encounter model; REM

\section{Introduction}

Estimation of population sizes has been one of the main goals in wildlife monitoring surveys [1]. Well into the second half of the previous century, survey methods were limited to capture-recapture and distance sampling studies using live traps, direct observations or indirect signs (i.e. feces), to estimate densities and population sizes [2-5]. The introduction of modern camera traps in the 1990's provided new ways to estimate abundance and initiated a surge in new research [6]. Most camera trap studies between 2008 and 2013, however, still used capture-recapture methods to estimate densities [7,8], a method limited to species that are individually marked $[9,10]$.

For unmarked animal populations, camera trap studies increasingly make use of relative abundance-indices to make inferences [8]. Unfortunately, relative abundance-indices are not comparable between sites, habitats, different points in time and species, as detection probability is not constant $[11,12]$. Additionally, relative abundanceindices do not produce estimates of the absolute number of individuals that are required for management of endangered populations.

Presently, two camera trapping methods estimate absolute numbers of individuals: the abundance-induced heterogeneity model [13] and the Random Encounter Model [14]. However, both models still have their drawbacks when estimating absolute numbers of individuals in an area (from here on referred to as 'species area abundance').

Abundance-induced heterogeneity (AIH) models are based on an occupancy framework, in which binary survey data are gathered over repeated visits. The AIH model assumes that detection probability $(r)$ is species specific and is related to its abundance $N_{i}$. Therefore variation in species detection probability at a sampled site, or point, can be used to estimate its abundance. In the AIH model, the mean abundance of a species across all sampled sites is expressed as Mean point abundance or Poisson parameter Lambda $(\lambda)$.

Lambda is estimated by the maximum likelihood of the binomial probability of observing a certain detection history over a set of $T$ repeat visits, multiplied by the Poisson probability of the local presence of $K$ animals on the site [15]. The key assumption of the AIH model is that the spatial distribution of the animals across survey sites follows a prior distribution (Poisson) [13]. Initially, the AIH model was developed for point count surveys of migratory birds, but researchers have more recently applied it to camera trap surveys (e.g. Argus phaesants Argus argusianus [16], Siamese Firebacks Lophura diardi [17]).

The limitation of the AIH model lies in its inability to estimate the effective detection area sampled. Estimations of species area abundance have been limited to a summation of Lambda over sampled sites and effective detection area is substituted for species characteristics such as average home range size $[17,18]$. Substitution, however, does not

*Corresponding author: Rademaker M, Forest and Nature Conservation Program, Wageningen University, Wageningen, The Netherlands, Tel: +31317 480 100; E-mail: mark1.rademaker@wur.n!

Received September 03, 2017; Accepted November 04, 2017; Published November 30, 2017

Citation: Rademaker M, Rode-Margono EJ, Weterings MJA (2017) Estimation of Species Area Abundance from Point Abundance Data, Using Effective Detection Areas from Camera Traps. J Biodivers Endanger Species 5: 200. doi: 10.4172/2332-2543.1000200

Copyright: (c) 2017 Rademaker M, et al. This is an open-access article distributed under the terms of the Creative Commons Attribution License, which permits unrestricted use, distribution, and reproduction in any medium, provided the original author and source are credited. 
Citation: Rademaker M, Rode-Margono EJ, Weterings MJA (2017) Estimation of Species Area Abundance from Point Abundance Data, Using Effective Detection Areas from Camera Traps. J Biodivers Endanger Species 5: 200. doi: 10.4172/2332-2543.1000200

Page 2 of 6

account for potential overlapping home-ranges, nor does it guarantee that camera traps effectively sample the home-range of a species.

The Random Encounter Model (REM) [14] was the first method to estimate the density and abundance of unmarked populations by taking detection probability of camera traps into account. This potentially provides a robust way to estimate species area abundance [19]. Further adjustments to the REM in 2011 allowed researchers to estimate the effective detection area of a camera trap for different species [20]. However, as the method is based on an ideal gas model, cameras must be placed randomly in relation to the movement of the target species. This restricts the sampling designs suitable for REM [21], and limits its use for cryptic species with low detection probability, that often require non-random sampling designs in order to generate sufficient captures.

Our objective is to present a novel method to estimate species area abundance of unmarked species that combines the advantages of the AIH- and REM models and that is suitable for a variety of sampling designs. Specifically, our model combines the AIH model with estimating the effective detection area from camera traps.

\section{Materials and Methods}

\section{A model for species area abundance estimation from point abundance data}

Conceptually, our newly proposed species area abundance (SAA) model consists of 5 basic steps (Figure 1). (1) The effective detection area of the camera traps for the target species is determined, providing the area sampled by each trap. (2) Using presence-absence data from repeat surveys in an $\mathrm{AIH}$ model, the mean point abundance, i.e. the mean abundance of a species across all sampled sites, is estimated. (3) This estimate is extrapolated to species area abundance by multiplying the mean point abundance with the total number of sampling sites if the entire survey area was monitored by camera traps without overlap

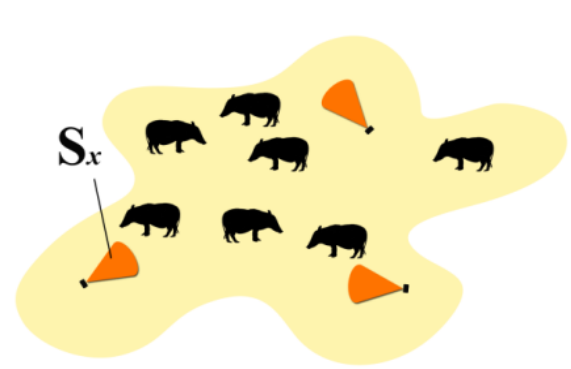

(a)

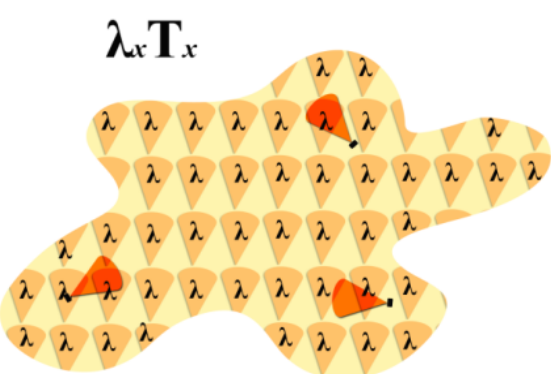

(c)

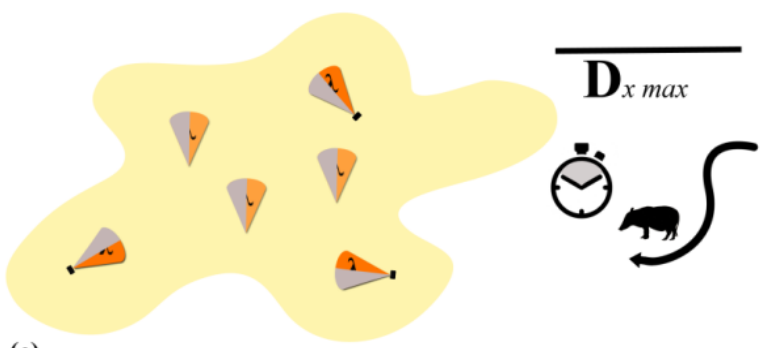

(e)

Figure 1: Schematic representation of the SAA model (a) a population is sampled using camera traps, with the effective area sampled by the camera traps indicated by the orange cones $\left(S_{x}\right)(b)$ based on detection histories, mean point abundance across sampling sites is estimated $(\lambda)$ (c) the estimate is extrapolated to area abundance by multiplying $\lambda$ with the total number of sampling sites as if the entire survey area was monitored by camera traps ( $\left.T_{x}\right)$ (d) the estimate is corrected for the total number of sites occupied, i.e. occupancy $\left(\psi_{x}\right)(\mathrm{e})$ finally, the estimate is corrected for animal activity and movement by multiplying the camera operating time by the proportion of time spent active by the target species and dividing this by the average time the species spends at each occupied locality, equal to the average length of each detection event. The result is the maximum number of detection events $\left(D_{x \max }\right)$. The estimate in (d) is divided by $D_{x \text { max }}$ to provide the number of animals in the area at any given time, i.e. species area abundance. 
between traps. (4) The extrapolated value is then corrected for the total number of sites occupied and (5) The resulting value is divided by the the maximum number of detections. The final estimate represents the number of individuals in the area at any given time, i.e. species area abundance. We will now explain the model in more detail.

\section{Effective detection area}

The effective detection area of a camera trap is related to both species body size and behaviour [20]. For species $x$, the effective detection area $\left(S_{x}\right)$ of a camera trap is equal to the segment of a circle that is the product of the species-specific effective detection radius $\left(r_{x}\right)$ and the species-specific effective detection angle $\left(\theta_{x}\right)[20]$.

$$
S_{x}=\frac{\theta_{x}}{2} r_{x}^{2} \quad(\theta \text { in radians })
$$

At the effective detection radius and angle, the expected number of detections for a species is equal to the expected number of detections missed [22]. Effective detection radius and angle are estimated from the detection radius and angle of an animal at first capture, using functions for fitting standard linear covariate detection models [20]. The threshold values at which the number of detections equals the number of detections missed can be computed using a line transect model for angle and a point transect model for radius in distance [20,23].

Within a single species, large differences between body size (e.g., between sex or life-stages) should be taken into account by estimating the radius and angle of detection for each sex or life-stage.

Furthermore, when using a combination of different camera trap models in the same survey or project, the effective detection area has to be (i.e. effective detection radius and angle) estimated for each camera trap model. The effective detection area should then be weighted based on the contribution of each camera trap model to the total number of traps used.

The maximum number of sampling sites without overlap $\left(T_{x}\right)$ for a species equals the size of the survey area $(A)$ divided by the effective detection area.

$$
T_{x}=\left(\frac{A}{S_{x}}\right)
$$

Mean point abundance: Mean point abundance $\left(\psi_{x}\right)$ can be estimated from the Royle-Nichols abundance-induced heterogeneity model [13].

Species area abundance: Species area abundance $\left(N_{x}\right)$ can be calculated by multiplying the mean point abundance by the proportion of sites occupied $\left(\psi_{x}\right)$ and the maximum number of local sampling sites.

$$
N_{x}=\lambda_{x} \psi_{x} T_{x}
$$

Equation 3 overestimates species abundance in an area, as it assumes a species to be present $24 \mathrm{~h}$ a day in each local sampling site. The result of equation 3 , therefore, needs to be corrected for the maximum number of times a camera could detect an individual of a species.

Proportion of sites occupied: The proportion of sites occupied $\left(\psi_{x}\right)$ can be estimated using single season occupancy models. Site covariates deemed important on the basis of the ecology of the target species should be included and several models compared. The total number of sites occupied is estimated as the sum of the mean of the posterior distribution of occupancy at each site and then divided by the total number of sites sampled [24].

\section{Maximum number of detections}

The maximum number of times a camera can detect an individual of a species depends on the average time the camera traps are operated $(t)$, the time a species spends at a local sampling site, and its level of activity throughout the day. If activity is defined as an animal being in movement [25], then the total amount of time in which detections could occur is equal to the total time the camera traps are operated, multiplied by the proportion of time spent active by the animal $\left(v_{x}\right)$. A flexible circular distribution to time-of-detection from camera trap data can be used to estimate the proportion of time spent active [25]. This method has two assumptions: the level of activity is the only determinant of the rate at which the camera detects animals i.e., the camera operating times and animal activity times are independent of one another, and all individuals in the sampled population are active at the peak of the daily activity cycle. If these assumptions are met, trap rate is proportional to the level of activity and the total amount of time spent active proportional to the area under the trap rate curve.

The proportion of time spent active multiplied by the average time the cameras are operated gives the maximum operating time $\left(v_{x} \bar{t}\right)$ available to detect a species. This maximum operating time, however, does not provide information on how many detection events could have occurred.

To calculate the maximum number of detection events, we must first assume that the presence of the camera traps does not alter the natural behaviour of the species. Secondly, we must assume that the local sites sampled are a representative sample of the habitats available in the area. If these assumptions are met, then the maximum number of detection events $\left(D_{x \max }\right)$ equals the maximum operating time divided by the average time a species spent at each local sampling site per detection event $\left(\bar{R}_{x}\right)$

$$
D_{x \max }=\frac{v_{x} \bar{t}}{\bar{R}_{x}}
$$

The use of equation 4 requires that the camera sensors are setup to allow continuous detection, either through the use of video recordings or the use of photo recordings without intervals. To identify gaps in time when camera traps do not function during the research period, camera traps should be set to take a picture every day at midnight.

Species area abundance can now be formulated as the product of the mean point abundance, the proportion of sites occupied and the maximum number of local sampling sites divided by the maximum number of detection events.

Species area abundance (SAA) model: $N_{x}=\frac{\lambda_{x} \psi_{x} T_{x}}{D_{x \max }}$

Dividing the extrapolated abundance estimates by the maximum number of detection events thus provides a 'snapshot' or 'frozen-intime' view of the number of animals in the area, similar to Distance sampling methods [26].

The SAA model assumes that all sampled local sites are accessible, animals are distributed homogenously over the habitat that they use, and abundance is constant for the duration of the survey. We recognize that this assumption might not be met in all cases. Stratified sampling within an animal's habitat can be an option if animals are clearly using specific parts of their habitat. Additionally, more cameras at random 
Citation: Rademaker M, Rode-Margono EJ, Weterings MJA (2017) Estimation of Species Area Abundance from Point Abundance Data, Using Effective Detection Areas from Camera Traps. J Biodivers Endanger Species 5: 200. doi: 10.4172/2332-2543.1000200

Page 4 of 6

locations are required to estimate density of species that are more aggregated or systematically distributed over their habitat. Next to this, abundance is assumed constant during the survey period. Therefore, repeated surveys need to be conducted in a sufficiently short timeperiod to ensure population closure.

\section{Case study bawean warty pigs}

We applied the SAA model to a camera-trapping dataset of Bawean warty pigs (Sus blouchi) from Bawean Island, Indonesia. Details of the random sampling design, population size estimates using REM modelling and an estimate of occupancy from these data can be found in Rademaker et al. [27]. Point abundance $(n=102)\left(\lambda_{x}\right)$ was computed in PRESENCE from the Royle-Nichols model [28]. We used a Chi-Square $\left(\chi^{2}\right)$ Goodness-Of-Fit test to assess whether the Poisson distribution fitted the dataset at the 0.05 significance level [29]. Each 24 hour period of a single operating camera represented a repeated survey. Each camera trap operated for 7 days, resulting in 7 repeated surveys. Data on detection radius and angles were collected in the field using a video viewer, compass and measuring tape. Camera trap videos were played on the video viewer and a field assistant positioned him/herself at the first detection point. A compass was then laid on the center of the camera trap to measure the angle of detection. This procedure was then repeated using a measuring tape to estimate detection radius. Effective detection radius and angle were estimated by DISTANCE 6.0 using a point-transect model for radius, and a line-transect model for angle data [23]. Propagation of error approach was used to estimate the uncertainty of species area abundance [30]. Uncertainty of the species area abundance estimate $N_{x}$ is equal to the square root of the squared sums of the uncertainty of the parameters $\lambda_{x}, T_{x}$ and $D_{x \max }$, times the partial derivatives of these parameters

$$
\sigma N_{x}=\sqrt{\left(\frac{\partial N_{x}}{\partial \lambda_{x}}\right)^{2} \sigma \lambda_{x}{ }^{2}+\left(\frac{\partial N_{x}}{\partial T_{x}}\right)^{2} \sigma T s_{x}{ }^{2}+\left(\frac{\partial N_{x}}{\partial D_{x \max }}\right)^{2} \sigma D_{x \max }^{2}}
$$

Uncertainty of the parameters $T_{x}$ and $D_{x \max }$ were themselves functions of uncertainty of the parameters $\theta_{x}, r_{x}$ and $t$ and $R_{x}$ (eqn 8) respectively.

$$
\begin{aligned}
& \sigma T_{x}=\sqrt{\left(\frac{\partial T_{x}}{\partial \theta_{x}}\right)^{2} \sigma \theta_{x}^{2}+\left(\frac{\partial T_{x}}{\partial r_{x}}\right)^{2} \sigma r_{x}^{2}} \\
& \sigma D_{x \max }=\sqrt{\left(\frac{\partial D_{x \max }}{\partial t}\right)^{2} \sigma t^{2}+\left(\frac{\partial D_{x \max }}{\partial R_{x}}\right)^{2} \sigma R_{x}^{2}}
\end{aligned}
$$

We used a two-sample t-test for equal means [29], to determine whether there was a significant difference between abundance estimates of the SAA-and REM model.

\section{Results}

\section{Species area abundance (SAA) Model}

Bawean warty pigs were detected at 45 out of 102 sites with an average of $(\bar{x} \pm \mathrm{SE}) 0.73 \pm 1.04$ detection events per site over the repeat surveys. Mean point abundance was estimated at $1.06 \pm 0.32$ pigs. Chisquare Goodness-Of-Fit test showed no significant difference between

\begin{tabular}{|c|c|c|c|c|}
\hline \multirow[b]{2}{*}{ Survey No. } & \multicolumn{4}{|c|}{ Parameters } \\
\hline & $\begin{array}{c}\text { No. of. } \\
\text { Detections }\end{array}$ & $\begin{array}{c}\text { No. of } \\
\text { Observed }\end{array}$ & $\begin{array}{c}\text { No. of } \\
\text { Expected }\end{array}$ & Chi-square ${ }^{*}$ \\
\hline \multirow[t]{3}{*}{1} & 0 & 96 & 91.4178 & 0.2297 \\
\hline & 1 & 6 & 10.0132 & 1.6085 \\
\hline & $>1$ & 0 & 0.5734 & 0.0000 \\
\hline \multirow[t]{3}{*}{2} & 0 & 89 & 91.4178 & 0.0639 \\
\hline & 1 & 13 & 10.0132 & 0.8909 \\
\hline & $>1$ & 0 & 0.5734 & 0.0000 \\
\hline \multirow[t]{3}{*}{3} & 0 & 92 & 91.4178 & 0.0037 \\
\hline & 1 & 10 & 10.0132 & 0.0000 \\
\hline & $>1$ & 0 & 0.5734 & 0.0000 \\
\hline \multirow[t]{3}{*}{4} & 0 & 88 & 91.4178 & 0.1278 \\
\hline & 1 & 14 & 10.0132 & 1.5874 \\
\hline & $>1$ & 0 & 0.5734 & 0.0000 \\
\hline \multirow[t]{3}{*}{5} & 0 & 93 & 91.4178 & 0.0274 \\
\hline & 1 & 9 & 10.0132 & 0.1025 \\
\hline & $>1$ & 0 & 0.5734 & 0.0000 \\
\hline \multirow[t]{3}{*}{6} & 0 & 91 & 91.4178 & 0.0019 \\
\hline & 1 & 11 & 10.0132 & 0.0972 \\
\hline & $>1$ & 0 & 0.5734 & 0.0000 \\
\hline \multirow[t]{3}{*}{7} & 0 & 91 & 91.4178 & 0.0019 \\
\hline & 1 & 11 & 10.0132 & 0.0972 \\
\hline & $>1$ & 0 & 0.5734 & 0.0000 \\
\hline \multicolumn{2}{|c|}{ Survey total } & 714 & 714.0308 & 4.84 \\
\hline pooled & & 0 & 0.569 & 0.569 \\
\hline \multicolumn{3}{|c|}{${ }^{*} \mathrm{df}=15, \mathrm{p}=0.99$, Critical $\mathrm{X}^{2}=7.26$} & & \\
\hline
\end{tabular}
observed and expected values, indicating model fit (Table 1). Mean parameter estimates to calculate species area abundance can be found
Table 1: Goodness-Of-Fit test results for mean point abundance in presence.

\begin{tabular}{|c|c|c|c|c|}
\hline Parameter & Mean & N & S.E. & S.E.\% \\
\hline$\lambda_{x}$ & 1.06 & 102 & 0.32 & 30.28 \\
\hline$\psi_{x}$ & 0.58 & 92 & & \\
\hline$T_{x}$ & 15631461 & & 0.35 & 0.00 \\
\hline$A$ & 46.6 & & & \\
\hline$r$ & 0.0039 & 63 & 0.00029 & 7.44 \\
\hline$\theta$ & 22.46 & 62 & 0.033 & 0.15 \\
\hline$D_{x \max }$ & 22093 & & 2507.28 & 11.35 \\
\hline$t$ & 596908 & 92 & 6539 & 1.10 \\
\hline$R$ & 15.67 & 57 & 1.77 & 11.30 \\
\hline$v$ & 0.58 & & & \\
\hline
\end{tabular}

Table 2: Mean parameter estimates and standard error of mean point abundance $\left(\lambda_{x}\right)$, proportion of sites occupied $\left(\psi_{x}\right)$, maximum number of local sampling sites $\left(T_{x}\right)$, survey area in $\mathrm{km}^{2}(\mathrm{~A})$, effective detection radius $(\mathrm{r})$, effective detection angle $(\theta)$ maximum number of detection events $\left(D_{x \max }\right)$, average camera operating time in seconds $(\bar{t})$, average time per detection event in seconds $(\bar{R})$ and activity level (v). Mean parameter estimates and standard error of $r, \theta, \psi$ and $v$ were obtained from Rademaker et al.

in Table 2. Species area abundance of Bawean warty pigs on Bawean Island was estimated to be $436 \pm 141$ individual pigs $(\bar{x} \pm \mathrm{SE})$ or $9.4 \pm$ 3.0 pigs per $\mathrm{km}^{2}$. Uncertainty of the SAA model estimate was $32.34 \%$.

\section{Random encounter model (REM)}

Rademaker et al. [27] estimated density and total abundance of Bawean warty pigs with the same dataset, although using a REM. Density and total abundance were estimated in two ways: one estimate with mean group size as an upper limit estimate, and one estimate without group size as a lower limit estimate. The lower limit estimate equalled $3.7 \pm 0.9 \mathrm{pigs} / \mathrm{km}^{2}$ or $172 \pm 42$ pigs on the whole island. The upper limit estimate was $8.1 \pm 1.9 \mathrm{pigs} / \mathrm{km}^{2}$ or $377 \pm 92$ pigs on the whole island. The uncertainty in the total abundance estimate by the REM was 
comparable to that obtained by the SAA model, however, slightly lower with $24.32 \%$. The density estimate per $\mathrm{km}^{2}$ obtained through the SAA model is significantly $(t(116.48)=-1.82, p=0.036)$ different to that of the lower limit estimate by the REM, but not significantly $(t(170.96)=-0.36$, $p=0.359)$ different to that of the upper limit estimate by the REM.

\section{Discussion}

\section{Model uncertainty}

We estimated species area abundance of Bawean warty pigs on Bawean Island by using mean point abundance from an abundanceinduced heterogeinity model and effective camera trap detection areas. The result is credible, although the uncertainty in the estimation by the SAA model is high, with mean point abundance $\left(\lambda_{x}\right)$, as the highest contributing parameter. Neither Royle and Nichols [13] nor O'Brien and Kinnaird [16] explicitly mention the uncertainty of mean point abundance for species assessed, although, the latter graphically report $95 \%$ CI of the estimates. Suwanrat et al. [17], report point abundance estimates of $0.49 \pm 0.13$ individuals $(\bar{x} \pm \mathrm{SE})$, equal to an uncertainty of $26.53 \%$.

When looking at uncertainties in REM estimates there is large variation in the literature. A study on Baird's tapir (Tapirus bairdii) yielded an average uncertainty of 54\% [31] and an average uncertainty of $39 \%$ was reported for Harvey's duiker (Cephalophus harveiyi) based on six differrent locations [32]. On the contrary an uncertainty of only $8 \%$ was reported in a study on European wildcats (Felis silvestris silvestri) [33] and an average uncertainty of $15 \%$ in a study of female African lionesses (Panthera leo spp.) in four habitats [34]. This shows the difficulty in defining an acceptable level of uncertainty in estimating abundance of unmarked species from camera trap data.

\section{Model comparison}

The abundance-induced heterogeneity model uses a Poisson distribution, and thus assumes that the spatial distribution of animals is homogeneous over the habitats [15]. In order to meet this assumptionthe number of animals inhabiting one sampling point should not be spatially correlated to the number of animals at other sampling points. This can be achieved by placing traps at a distance greater than the home range diameter of the focal species when using non-random sampling designs. Additionally, the abundance-induced heterogeneity model assumes that in order to accurately estimate the maximum number of detection events, sampled points are a representative sample of the habitats available in the area. These assumptions allow for non-random and random sampling designs.

The REM is derived from an ideal gas model in which particles are assumed to move randomly in relation to one another and the number of collissions or density of particles in the gas can be calculated based on this assumption. The key requirement for calculating animal density using the REM model is thus that the placement of the camera traps must be random in relation to the movement of the animals. Within habitats sampled by the researcher (e.g., secondary forest), landscape features that are used or avoided by the target species more than proportionally (e.g., trails, places with scratchmarks or feces), must therefore only be sampled in proportion to their coverage in the landscape to prevent violation of model assumptions [21]. Random (stratified) or systematicinterval sampling designs can meet this assumption [35,36]. Use of random or systematic sampling design makes the use of the REM limited for cryptic species whose detection probability is low and who disproportionally use certain landscape features. In that case, locally preferrential placement is the only option to obtain any detection event or to get a sufficient amount of observations to accurately estimate abundance as well as effective detection parameters.

The abundance induced heterogeneity model and the SAA model described in this paper do not have this limitation as they do not rely on trapping rate, but presence-absence data. An additional capture within a repeat survey resulting from a non-random movement of the target species in relation to a camera trap's position does not directly influence the abundance estimate as the number of presence detections during the survey is still 1 . Available habitats must be sampled representatively, but at these sampling sites locally optimal locations within a certain radius (e.g., $100 \mathrm{~m}$ ), such as trails, places with tracks, feces or other signs of recent activity, can be sampled and representative estimates of abundance can still be obtained [16]. This makes these models more suitable to estimate species area abundance of more rare or cryptic species. Repeated surveys do need to be conducted in a sufficiently short time-period to meet the assumption of population closure. Further studies are needed to determine the level of accuraccy and practicality of the SAA model for different species and sampling designs.

\section{Conclusion}

We used the newly developed Species Area Abundance (SAA) model to estimate the abundance of Bawean warty pigs on Bawean. The standard error of estimated abundance on Bawean was slightly greater than the standard error of abundance estimated by an REM model used for comparison, but lay within the range of uncertainties of a number of other REM studies. An advantage of the SAA model in studying abundances of rare or cryptic species in an area are the less restrictive assumptions in terms of sampling design. Further studies are needed to determine the accuracy and practicality of species area abundance estimations under different sampling designs.

\section{Acknowledgements}

We would like to thank Erik Meijaard for reviewing an early version of the manuscript. Next to this, we would like to thank the anonymous reviewer whose comments helped to further improve and clarify the manuscript during the submission process

\section{References}

1. Wight HM (1938) A Manual on Field and Laboratory Technic in Wildlife Management. University of Michigan Press, Ann Arbor, USA.

2. Caughley G (1977) Analysis of Vertebrate Populations. John Wiley and Sons, New York City, New York, USA

3. Burnham KP, Anderson DR, Laake JL (1980) Estimation of density from line transect sampling of biological populations. Wildlife monographs 72: 3-202.

4. White GC (1982) Capture-recapture and removal methods for sampling closed populations. Los Alamos National Laboratory.

5. Bailey RE, Putman RJ (1981) Estimation of fallow deer (Dama dama) populations from faecal accumulation. J Appl Ecol 18: 697-702.

6. O‘Connell AF, Nichols JD, Karanth KU (2010) Camera traps in animal ecology: methods and analyses. Springer Science \& Business Media, USA.

7. Foster RJ, Harmsen BJ (2012) A critique of density estimation from cameratrap data. The Journal of Wildlife Management 76: 224-236.

8. Burton AC, Neilson E, Moreira D, Ladle A, Steenweg R et al. (2015) Review: Wildlife camera trapping: a review and recommendations for linking surveys to ecological processes. J Appl Ecol 52: 675-685

9. Soisalo MK, Cavalcanti SM (2006) Estimating the density of a jaguar population in the Brazilian Pantanal using camera-traps and capture-recapture sampling in combination with GPS radio-telemetry. Biol Conserv 129: 487-496.

10. Karanth KU, Nichols JD, Kumar N, Hines JE. (2006) Assessing tiger population dynamics using photographic capture-recapture sampling. Ecology 87: 29252937 
Citation: Rademaker M, Rode-Margono EJ, Weterings MJA (2017) Estimation of Species Area Abundance from Point Abundance Data, Using Effective Detection Areas from Camera Traps. J Biodivers Endanger Species 5: 200. doi: 10.4172/2332-2543.1000200

11. Harmsen BJ, Foster RJ, Silver S, Ostro L, Doncaster CP (2010) Differential use of trails by forest mammals and the implications for camera-trap studies: a case study from Belize. Biotropica 42: 126-133.

12. Sollmann, R, Gardner B, Chandler RB, Shindle DB, Onorato DP et al. (2013) Using multiple data sources provides density estimates for endangered Florida panther. J Appl Ecol 50: 961-968.

13. Royle JA, Nichols JD (2003) Estimating abundance from repeated presenceabsence data or point counts. Ecology 84: 777-790.

14. Rowcliffe JM, Field J, Turvey ST, Carbone C (2008) Estimating animal density using camera traps without the need for individual recognition. J Appl Ecol 45: 1228-1236.

15. Donovan TM, Hines J (2007) Exercises in occupancy estimation and modeling estimation. Vermont Cooperative Fish and Wildlife Research Unit Spreadsheet Project, USA.

16. O‘Brien TG, Kinnaird MF (2008) A picture is worth a thousand words: the application of camera trapping to the study of birds. Bird Conserv Int 18 S144-S162.

17. Suwanrat S, Ngoprasert D, Sutherland C, Suwanwaree P, Savini T (2015) Estimating density of secretive terrestrial birds (Siamese Fireback) in pristine and degraded forest using camera traps and distance sampling. Global Ecol Conserv 3: 596-606.

18. Karanth KU, Nichols JD (1998) Estimation of tiger densities in India using photographic captures and recaptures. Ecology 79: 2852-2862.

19. Jennelle CS, Runge MC, MacKenzie DI (2002) The use of photographic rates to estimate densities of tigers and other cryptic mammals: a comment on misleading conclusions. Anim Conserv 5: 119-120.

20. Rowcliffe J, Carbone C, Jansen PA, Kays R, Kranstauber B (2011) Quantifying the sensitivity of camera traps: an adapted distance sampling approach. Methods Ecol Evol 2: 464-476.

21. Rowcliffe JM, Kays R, Carbone C, Jansen PA (2013) Clarifying assumptions behind the estimation of animal density from camera trap rates. J Wild Manage 77: 876-876.

22. Buckland ST, Anderson DR, Burnham KP, Laake JL (1993) Statistical Theory In: Distance Sampling: Estimating Abundance of Biological Populations (pp: 52-99). Springer-Science+Business Media, BV, Netherlands.

23. Thomas L, Laake JL, Rexstad E, Strindberg S, Marques FFC, et al. (2009) Distance 6.0. Releas " $x$ "1. Research Unit for Wildlife Population Assessment University of St. Andrews, United Kingdom.
24. Fiske I, Chandler R (2011) unmarked: An R package for fitting hierarchical models of wildlife occurrence and abundance. J Stat Softw 43: 1-23.

25. Rowcliffe JM, Kays R, Kranstauber B, Carbone C, Jansen PA (2014) Quantifying levels of animal activity using camera trap data. Methods Ecol Evol 5: 1170-1179.

26. Howe EJ, Buckland ST, Després-Einspenner ML, Kühl HS (2017) Distance sampling with camera traps. Methods Ecol Evol 8: 1558-1565.

27. Rademaker M, Meijaard E, Semiadi G, Blokland S, Neilson EW, et al. (2016) First Ecological Study of the Bawean Warty Pig (Sus blouchi), One of the Rarest Pigs on Earth. Plos one 11: e0151732.

28. Hines JE (2006) PRESENCE2- Software to estimate patch occupancy and related parameters.

29. Snedecor GW, Cochran WG (1989) Statistical Methods. Eighth Edition, lowa State University Press, USA.

30. Taylor JR (1997) Propagation of Uncertainties. In: An Introduction to Erro Analysis: The Study of Uncertainties in Physical Measurements ( $2^{\text {nd }}$ edition) University Science Books, Sausolito, USA, pp: 45-73.

31. Carbajal-Borges JP, Godínez-Gómez O, Mendoza E (2014) Density, abundance and activity patterns of the endangered Tapirus bairdii in one of its last strongholds in southern Mexico. Trop Conserv Sci 7: 100-114.

32. Rovero F, Marshall AR (2009) Camera trapping photographic rate as an index of density in forest ungulates. J Appl Ecol 46: 1011-1017.

33. Anile S, Ragni B, Randi E, Mattucci F, Rovero F (2014) Wildcat population density on the Etna volcano, Italy: a comparison of density estimation methods. J Zool 293: 252-261

34. Cusack JJ, Swanson A, Coulson T, Packer C, Carbone C, et al. (2015) Applying a random encounter model to estimate lion density from camera traps in Serengeti National Park, Tanzania. J Wildl Manage79: 1014-1021.

35. Kays R, Tilak S, Kranstauber B, Jansen PA, Carbone C, et al. (2010) Monitoring wild animal communities with arrays of motion sensitive camera traps. arXiv preprint arXiv:1009.5718.

36. Ahumada JA, Silva CE, Gajapersad K, Hallam C, Hurtado J, et al. (2011) Community structure and diversity of tropical forest mammals: data from a global camera trap network. Philos Trans R Soc Lond B Biol Sci 366: 27032711. 